\title{
Demonstration of Immune Complex Deposits Using Fluorescence Microscopy of Hematoxylin and Eosin- Stained Sections of Hollande's Fixed Renal Biopsies
}

\author{
James T. McMahon, Ph.D., Jonathan L. Myles, M.D., Raymond R. Tubbs, D.O. \\ Department of Anatomic Pathology, Cleveland Clinic Foundation, Cleveland, Ohio
}

\begin{abstract}
A new method that may be useful in the evaluation of renal biopsies is described using fluorescence microscopy on standard hematoxylin and eosinstained sections of kidney tissue fixed in Hollande's fixative. We describe brightly fluorescing immune complex deposits within glomerular basement membranes and mesangial matrices that correlate well with the results of standard direct immunofluorescence on frozen tissue and electron microscopy. In a blind analysis of 261 consecutive renal biopsies, we determine that this method has diagnostic utility for identification of immune complex glomerulonephritis and significantly extends the usefulness of standard histologic preparations before the use of special stains or procedures.
\end{abstract}

KEY WORDS: Fluorescence, H\&E, Hollande's fixative, Immune complex, Kidney.

Mod Pathol 2002;15(9):988-997

Demonstration of immune complex deposits is necessary for proper classification of renal disease. With combined use of light microscopy, immunofluorescence, and electron microscopy (EM), deposits can be immunologically characterized and accurately located within the nephron. For light microscopy, hematoxylin and eosin (H\&E) and periodic acid-Schiff are routinely used for general morphology. Special stains including Jones silver methenamine (1) may be used to infer the location of deposits by demonstrating "spikes" and defects in glomerular basement membranes. Other special stains have been reported to demonstrate deposits themselves (2-6). Although these stains are useful and may be part of a routine protocol, they involve

Copyright $(2002$ by The United States and Canadian Academy of Pathology, Inc.

VOL. 15, NO. 9, P. 988, 2002 Printed in the U.S.A.

Date of acceptance: May 20, 2002.

Address reprint requests to: James T. McMahon, Ph.D., Department of Anatomic Pathology, Cleveland Clinic Foundation, 9500 Euclid Avenue, Cleveland, OH 44195; e-mail: mcmahojt@ccf.org; fax: 216-445-6967.

DOI: 10.1097/01.MP.0000027202.51385.85 additional time and expense that may be unnecessary, and their results are not as definite as those of immunofluorescence and EM. We recently discovered that routine H\&E-stained sections of kidney biopsies fixed in Hollande's fixative, when viewed with fluorescence microscopy (H\&E/FM), show immune complex deposits in both mesangial and glomerular capillary wall locations that are readily distinguishable from surrounding cellular and matrix components. We describe here the appearance of immune complex deposits in renal biopsies using $\mathrm{H} \& \mathrm{E} / \mathrm{FM}$ and report the diagnostic utility of H\&E/FM in a series of 261 renal biopsies in which $\mathrm{H} \& \mathrm{E} / \mathrm{FM}$ was performed and evaluated without prior knowledge of the results of standard direct immunofluorescence and EM.

\section{MATERIALS AND METHODS}

\section{Tissue Preparation and Microscopy}

All renal biopsies were processed according to a standard protocol used at the Cleveland Clinic Foundation. Biopsies were received and examined at the surgical desk for adequacy using a dissection microscope. Biopsies were divided into three portions with glomeruli identified for light, immunofluorescence, and electron microscopies. Each microscopic modality was used for complete characterization of the biopsy. Light-microscopic stains included H\&E and periodic acid-Schiff with selective use of Congo red. Immunofluorescence for IgG, IgA, IgM, C3c, albumin, and $\kappa$ and $\lambda$ light chains, when indicated, was performed on sections of frozen fresh tissue. Electron microscopy was performed on glutaraldehyde/osmium tetroxide-fixed tissue embedded in Spurr epoxy resin. Although each modality is considered essential for proper diagnosis of renal disease, only fluorescence microscopy of $H \& E$ sections (H\&E/FM) will be described here in detail. Results of immunofluorescence and EM are supplemented to verify the findings of $\mathrm{H} \& \mathrm{E} / \mathrm{FM}$. 
The portion for light microscopy was fixed in Hollande's fixative consisting of $3.7 \%$ formalin, $40 \%$ picric acid, $25 \%$ copper acetate, and $1.5 \%$ acetic acid for 2 hours to overnight. Hollande's fixative can be prepared as shown in Table 1 or obtained commercially from numerous scientific vendors. After fixation, tissue was thoroughly rinsed under a running tap until rinse water ran clear. Tissue was then processed overnight on a ThermoShandon autoprocessor (Pittsburgh, PA; 15275) using a standard schedule for dehydration and paraffin infiltration. Sections were taken at a thickness of 2-4 $\mu \mathrm{m}$ and stained in Harris' unacidified hematoxylin and an alcoholic solution of eosin $\mathrm{Y}$ (both obtained from ThermoShandon). Sections were coverslipped and examined using a Leitz photomicroscope equipped with a 100-W halogen lamp for brightfield microscopy and a 100-W mercury vertical illuminator with a 450- to 490-nm excitation filter and a $515-\mathrm{nm}$ suppression filter for fluorescence microscopy.

\section{Selection of Cases}

Cases were selected from archives to include normal kidney and a wide variety of kidney diseases including diabetic nephropathy, renal amyloidosis, focal segmental glomerulosclerosis, postinfectious glomerulonephritis (GN), membranoproliferative GN including Types I and II, lupus nephritis, crescentic GN including anti-GBM disease, mesangiopathic GN, membranous GN, cryoglobulinemic GN, fibrillary GN, minimal change disease, thin membrane disease, microangiopathy, and light chain deposition disease.

\section{Analysis of Method}

To determine whether H\&E/FM is helpful in the routine evaluation of medical renal disease, archived H\&E sections of 261 consecutive kidney biopsies from January 1997 to June 1998 were examined using H\&E/FM without knowledge of results of immunofluorescence or EM. Slides were examined using standard light and fluorescence microscopy. The ability to switch between brightfield light microscopy and fluorescence microscopy on the same $H \& E$ section allowed easy correlation of fluorescence patterns with well-recognized histologic and

TABLE 1. Preparation of Hollande's Fixative

\begin{tabular}{lc}
\hline \multicolumn{1}{c}{ Substance } & Quantity \\
\hline Distilled or deionized water & $850 \mathrm{~mL}$ \\
Copper acetate & $25 \mathrm{~g}$ \\
Picric acid & $40 \mathrm{~g}$ \\
Formalin, 37\% & $100 \mathrm{~mL}$ \\
Acetic acid & $15 \mathrm{~mL}$ \\
Distilled or deionized water & up to $1000 \mathrm{~mL}$ \\
\hline
\end{tabular}

Ingredients should be mixed and completely dissolved in order listed. pathologic renal structures. Glomeruli were scored for the presence and location of fluorescent deposits. Specimens not having obvious deposits using $20 \times$ or $40 \times$ objectives were routinely reexamined at oil immersion lens magnification to enhance fluorescence and permit detection of small deposits. This was found necessary for detection of subtle deposits such as those in Stage I membranous glomerulonephritis. Deposits and their location in mesangia and/or glomerular basement membranes were considered significant if fluorescence was strongly above surrounding matrix and scored positive if they resembled the recognizable patterns of standard direct immunofluorescence usually associated with glomerular immune complex disease. Findings were tabulated and subsequently compared with the diagnoses and descriptions in the final pathologic reports that served as the standard.

\section{Comparison of Fixatives}

At the Cleveland Clinic, Hollande's fixative is preferentially used in the histologic processing of most tissues including kidney because of superior nuclear detail $(7,8)$. Formalin, however, is a more widely used fixative and may differ in the mechanism by which specific chemical moieties are preserved in tissue. To determine the role of fixatives in the demonstration of immune complexes, H\&E/FM of kidney tissue fixed in Hollande's was compared with H\&E/FM of formalin-fixed kidney biopsies from six patients previously diagnosed as having membranous glō̄ērulonephritis.

\section{RESULTS}

When viewed by fluorescence microscopy, $H \& E$ sections of any tissue brightly fluoresce green or yellow-green against a dark background. This fluorescence is principally a function of the eosin component of the H\&E stain. Eosin, although widely used as a histologic stain for light microscopy, is itself a fluorochrome commercially produced by bromination of fluorescein to give tetrabromofluorescein (eosin Y).

With the addition of four bromine residues, eosin has the same heterocyclical ring structure as fluorescein, and retains the strong fluorescence property of its parent compound.

\section{Normal Kidney}

Histologic details of an H\&E section of normal kidney were comparable using both routine light microscopy (LM; Fig. 1A) and fluorescence microscopy (FM; Fig. 1B). Readily identified in both were glomerular, tubular, and vascular structures. In the fluorescence image (Fig. 1B), however, there was 

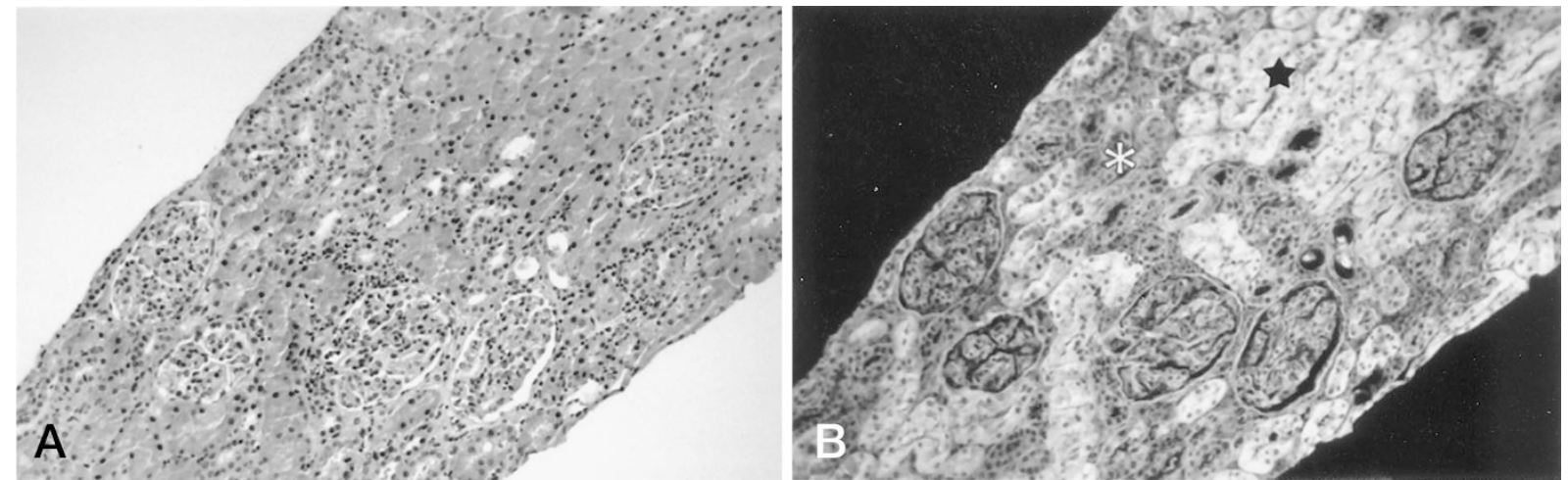

FIGURE 1. Comparison of routine light microscopy (LM; A) and fluorescence microscopy (B) of identical hematoxylin and eosin (H\&E) section of renal biopsy. Fluorescence microscopy of H\&E section (H\&E/FM) allows easy differentiation between proximal ( $\star$ ) and distal (*) convoluted tubules that is not apparent by LM. Glomeruli are also more prominently visualized by H\&E/FM $(90 \times)$.

better differentiation between proximal and distal tubules, and glomeruli were more distinct. By routine LM (Fig. 1A), proximal tubules, in general, were more granular and more eosinophilic than distal tubules. Because they were more eosinophilic, proximal tubules emitted greater fluorescence by FM (Fig. 1B) and in a more granular pattern than did distal tubules, making the two readily distinguishable using H\&E/FM. Also glomeruli were more readily recognized using $\mathrm{H} \& \mathrm{E} / \mathrm{FM}$ because individual glomerular segments were sharply accentuated against the darkness of Bowman's space (Fig. 1B). Cell nuclei are preferentially stained by hematoxylin rather than eosin. Therefore, positions occupied by nuclei were devoid of fluorescence with $\mathrm{H} \& \mathrm{E} / \mathrm{FM}$ and appeared/dark or vacant within the highly fluorescent domain of cytoplasm. Extracellular structures such as collagen and basement membrane were well defined by H\&E/FM but extracellular spaces not occupied by structured matrix appeared dark. Although most cells and matrix components fluoresced green or yellow-green, red blood cells had a yellow-orange tinge. Using $\mathrm{H} \& \mathrm{E} / \mathrm{FM}$ at high magnification and especially at oil immersion lens magnification (Fig. 2), glomerular basement membranes were viewed as thin and delicate with smooth uniform fluorescence. Mesangia showed consistent homogeneous fluorescence of the matrix component, whereas constituent mesangial cells corresponded to areas of increased fluorescence having a structured or granular appearance.

\section{Diseased Kidney}

Renal diseases may involve glomeruli, tubules, vasculature, and interstitium individually and in combination. H\&E/FM was not found significantly helpful in diseases of the kidney outside the glomerulus such as tubular, vascular, or interstitial disease but was especially useful in identifying glo- merular deposits associated with immune complex glomerulonephritis (ICGN). H\&E/FM of ICGN revealed glomerular deposits as particulate or globular areas of high fluorescence readily distinguishable from the lower fluorescence of surrounding cellular and matrix components. Specific patterns of fluorescence using H\&E/FM were frequently distinctive enough to predict the type of ICGN normally revealed only by subsequent immunofluorescence or EM. Cases of ICGN in which H\&E/FM proved particularly helpful in identifying immune complex deposits included membranous glomerulonephritis (MGN) including lupus nephritis (World Health Organization [WHO] Class V), immune complex-related mesangiopathic glomerulonephritis (MesGN), and certain types of membranoproliferative glomerulonephritis (MPGN).

\section{Membranous Glomerulonephritis/Lupus Nephritis (V)}

In our study, we-looked at both early and late stages of MGN using both routine LM and H\&E/ FM. By LM, glomeruli in Stage I-II MGN showed

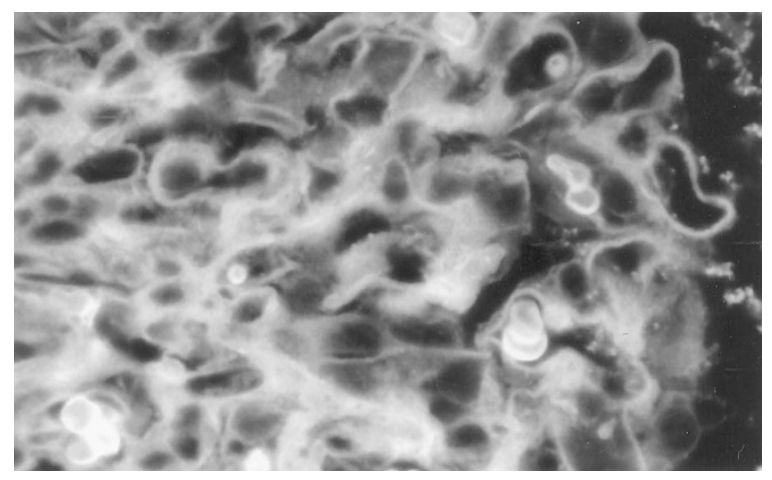

FIGURE 2. Hematoxylin-eosin stain, viewed by fluorescence microscopy, of normal kidney. Glomerular capillary loops are thin and delicate. Mesangial matrices show smooth, even fluorescence. Mesangial cells show increased, granular fluorescence $(460 \times)$. 

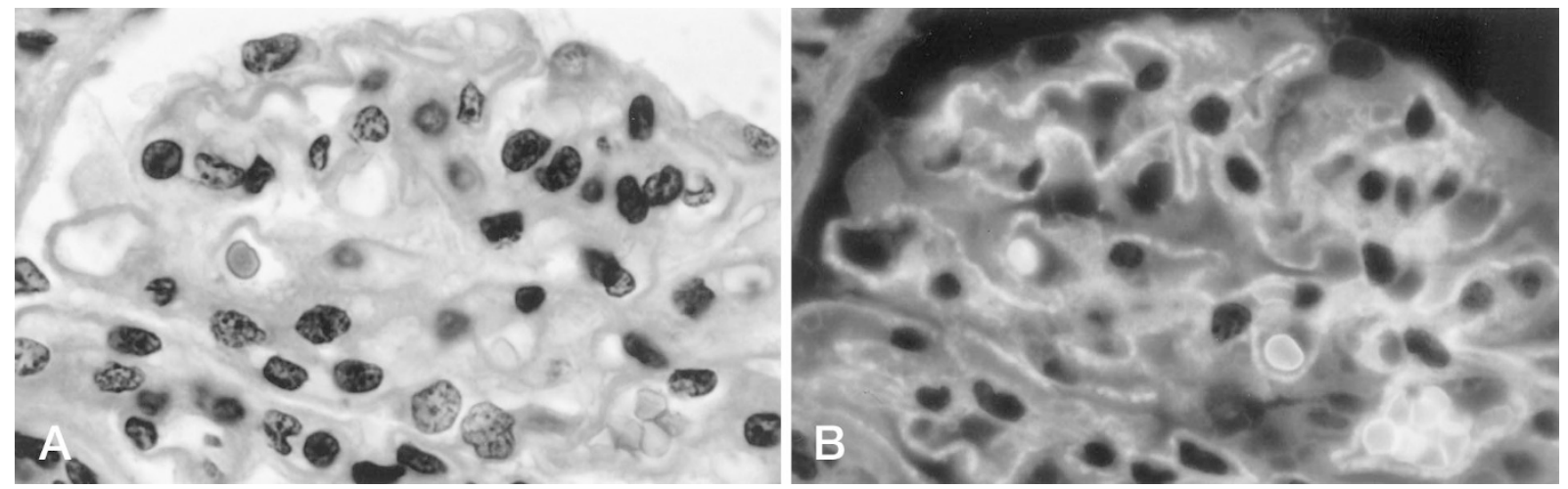

FIGURE 3. Comparison of routine light microscopy (LM; A) and fluorescence microscopy (B) of identical hematoxylin-eosin (H\&E)-stained section of early-stage membranous glomerulonephritis. By LM (A), glomerular capillary loops are slightly to moderately thickened. There is no evidence of immune complex deposits. By H\&E viewed by fluorescence microscopy (B), there are numerous fluorescent deposits appearing as beads on a string, corresponding to immune complex deposits along glomerular basement membranes $(460 \times)$.

minimal to moderate thickening of the GBM, but no other abnormality was evident (Fig. 3A). However, by H\&E/FM of the identical glomerulus, the GBM could be seen as having numerous linear series of small points of fluorescence appearing as beads on a string (Fig. 3B). The beads corresponded to immune complex deposits, whereas the string represented the GBM. Beads were strung closely together and were of variable size and may have had intervening gaps. This pattern and distribution of beads on a string was typical of MGN using H\&E/FM and agreed well with the pattern and distribution of deposits seen in the immunofluorescence (Fig. 4) and EM (Fig. 5) of the same case of MGN. In more advanced cases, as in MGN Stages II-III, the GBM was thicker and the deposits larger. By routine LM, thickened GBM was evident (Fig. $6 \mathrm{~A})$, but demonstration of deposits was lacking and would still have to wait for immunofluorescence or EM. By H\&E/FM of the same section (Fig. 6B), thickened GBM was again evident, but deposits were seen dramatically in advance of immunofluorescence and EM. Deposits were much larger than those of MGN I-II (Fig. 3B) and generally were separated by fewer gaps. Like MGN, the membranous variant of lupus glomerulonephritis (WHO Class V) showed nonspecific pathologic features such as thickened GBM by routine LM, whereas $\mathrm{H} \& \mathrm{E} / \mathrm{FM}$ revealed a distinct beads-on-a-string pattern of fluorescence (Fig. 7) corresponding to subepithelial dense deposits by EM.

\section{Mesangiopathic Glomerulonephritis}

In MesGN, frequently there is proliferation of cells and matrix in the mesangia with little involvement of the capillary walls or lumina. These changes may occur with or without the formation of immune complex deposits in the mesangia. $\mathrm{H} \& \mathrm{E} / \mathrm{FM}$ of MesGN with deposits revealed in- tensely fluorescent granular areas within the mesangial matrix and/or intense bands of condensed fluorescence adjacent to the free edges of the mesangia (Fig. 8). In our study, these fluorescent patterns frequently were unmistakable and readily associated with familiar immunofluorescence and EM of immune complex-related MesGN, including IgA nephropathy, Henoch-Schönlein purpura, mesangiopathic lupus nephritis, and minimal change disease with mesangial deposits. However, because

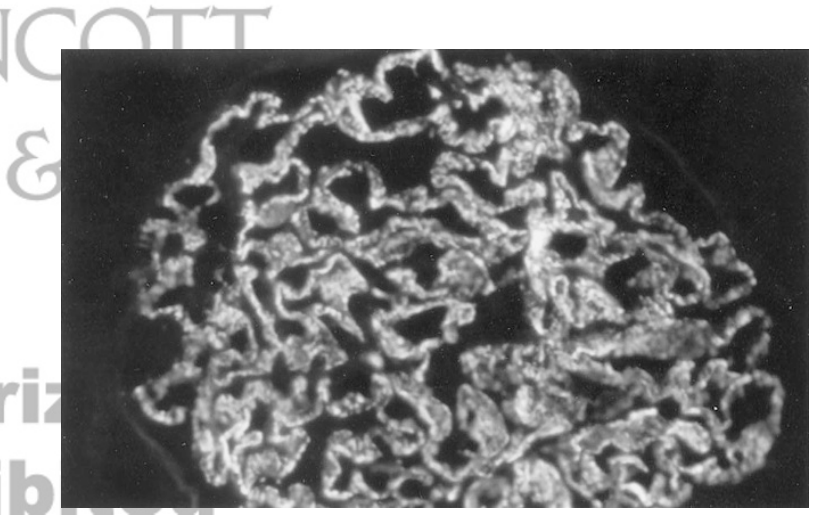

FIGURE 4. Immunofluorescence of same case illustrated in Figure 3, showing localization of immunoglobulin G deposits $(180 \times)$.

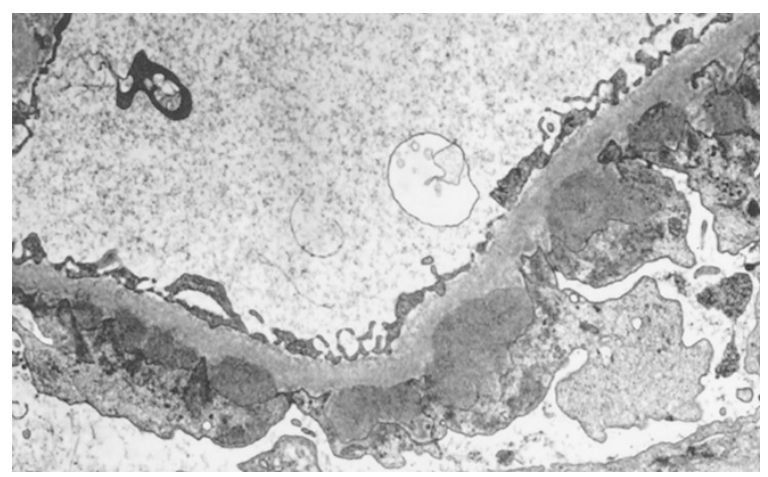

FIGURE 5. Electron microscopy of same case illustrated in Figures 3 and 4 , showing location of electron-dense deposits along glomerular basement membrane $(6300 \times)$. 

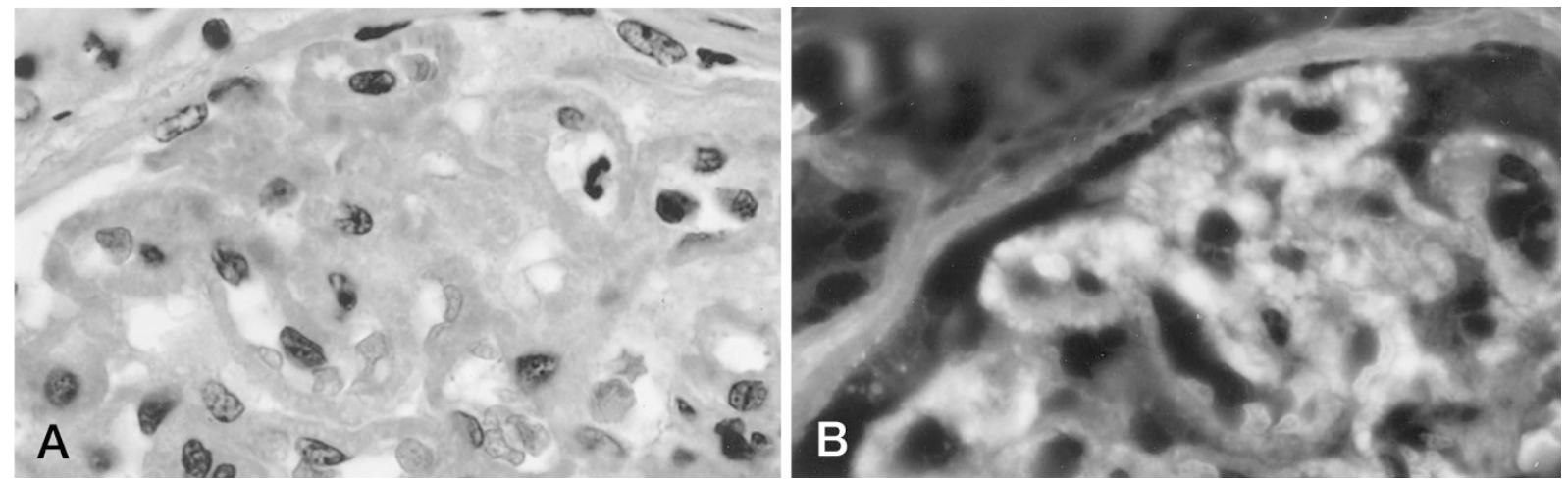

FIGURE 6. Comparison of routine light microscopy (LM; A) and hematoxylin-eosin stain (H\&E) viewed by fluorescence microscopy (H\&E/FM; B) of identical H\&E-stained section of late-stage membranous glomerulonephritis. By LM (A), glomerular capillary loops are greatly thickened, but there is no evidence of immune complex deposits. By H\&E/FM (B), there are large fluorescent deposits seen along glomerular basement membranes corresponding to immune complex deposits $(460 \times)$.

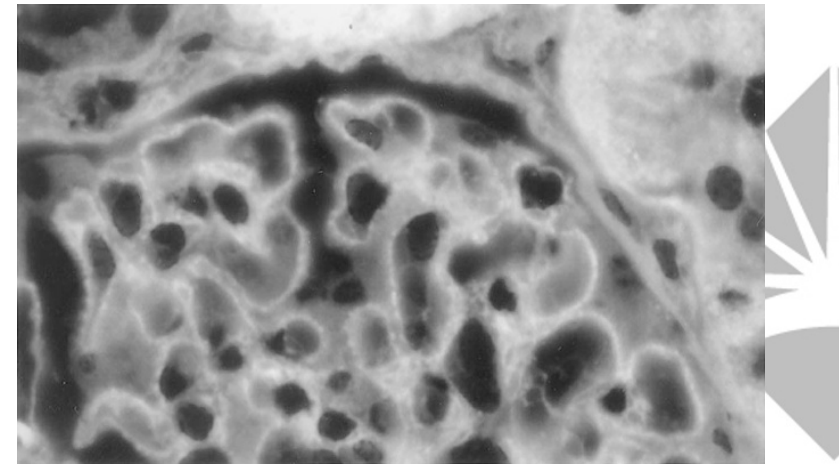

FIGURE 7. Hematoxylin-eosin stain, viewed by fluorescence microscopy, of lupus nephritis (WHO Class V) showing beads-on-astring pattern of fluorescence corresponding to immune complex deposits along glomerular basement membranes $(460 \times)$.

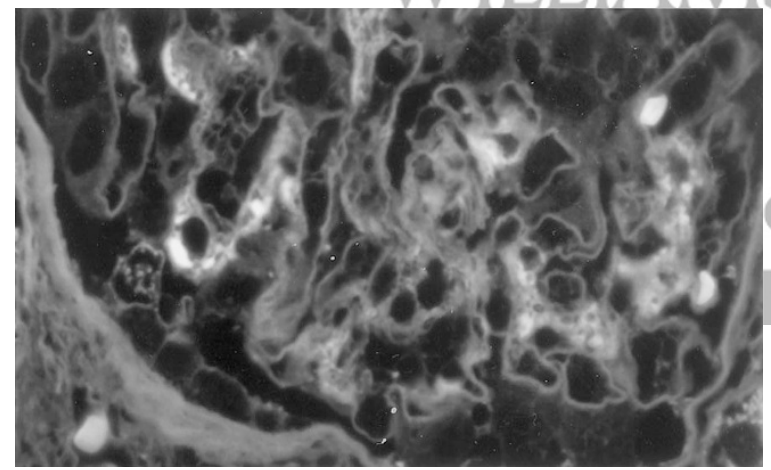

FIGURE 8. Hematoxylin-eosin stain, viewed by fluorescence microscopy, of section of mesangiopathic glomerulonephritis, immune complex type, showing fluorescent deposits in glomerular mesangia corresponding to immune complex deposits $(460 \times)$.

of small size, few deposits, or low intensity, some fluorescent deposits related to immune complexes in MesGN were difficult to differentiate from mesangial fluorescence coming from the mixed granularity and cellularity of the glomerular mesangia. In these cases, H\&E/FM of immune complex deposits in MesGN were unrecognized or equivocal.

\section{Membranoproliferative}

Glomerulonephritis/Lupus Nephritis (IV)

Mesangial proliferation and a marked increase in mesangial matrix along with thickening and splitting of peripheral capillary loops are wellrecognized features of MPGN (MPGN I), cryoglobulinemic $\mathrm{GN}$, and diffuse proliferative lupus nephritis (WHO Class IV). In this type of setting, positive fluorescence using H\&E/FM was anticipated and not difficult to identify. By H\&E/FM, glomerular capillary loop deposits appeared either granular or as continuous bands of fluorescence of variable thickness that followed the contour of peripheral glomerular capillary loops (Fig. 9). These deposits corresponded well to deposits seen by direct immunofluorescence (Fig. 10) and EM (Fig. 11). $\mathrm{H} \& \mathrm{E} / \mathrm{FM}$ also demonstrated fluorescent mesangial deposits, but as in MesGN, these deposits frequently were difficult to recognize.

Some cases were distinctive by H\&E/M because of the presence of immune complex deposits associated with a proliferation of inflammatory cells within glomeruli. In cases of postinfectious glomerulonephritis, routine LM showed a variable in-

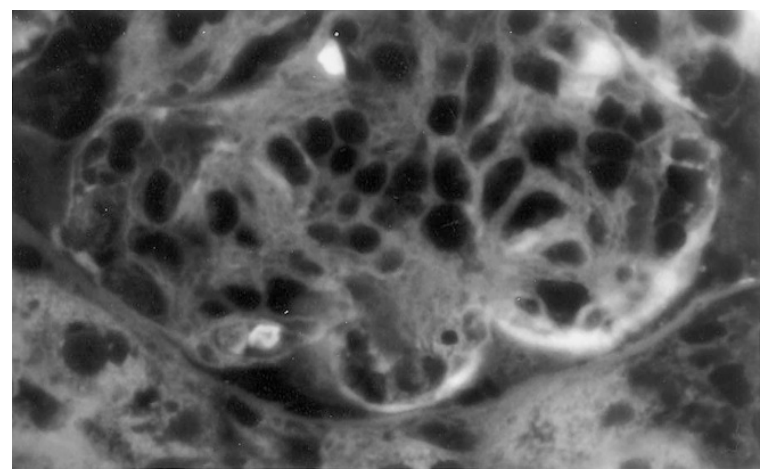

FIGURE 9. Hematoxylin-eosin stain, viewed by fluorescence microscopy, of membranoproliferative glomerulonephritis showing fluorescent deposits corresponding to immune complexes along the contour of glomerular basement membranes $(460 \times)$. 


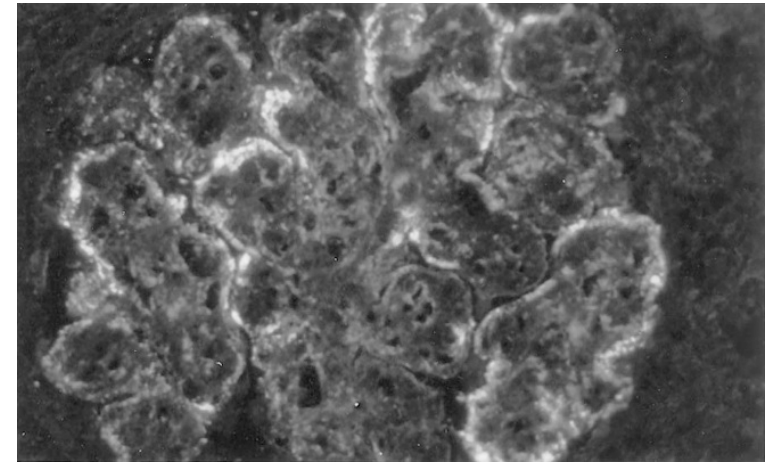

FIGURE 10. Immunofluorescence of same case of membranoproliferative glomerulonephritis illustrated in Figure 9 showing localization of immunoglobulin $\mathrm{G}$ in glomerular capillary loops $(180 \times)$.

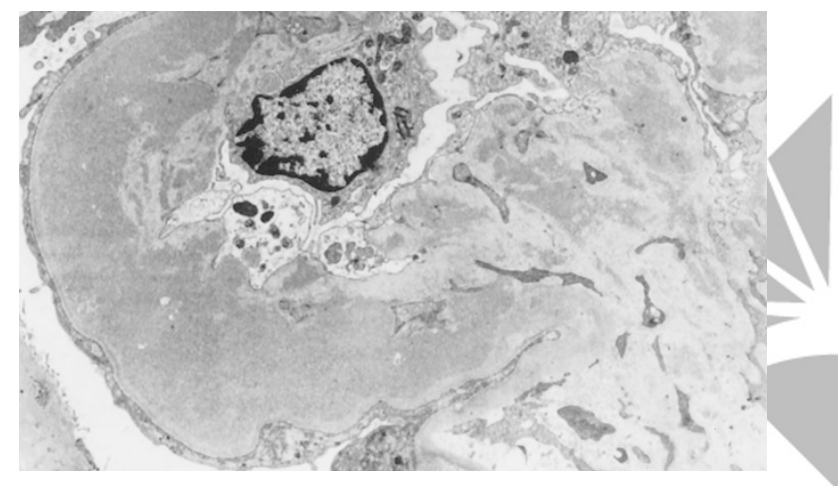

FIGURE 11. Electron microscopy of same case of membranoproliferative glomerulonephritis illustrated in Figures 9 and 10 showing location of large subendothelial electron-dense deposit along glomerular basement membrane $(4000 \times)$.

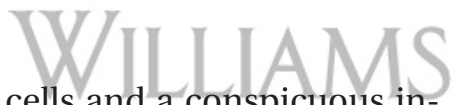

crease in endocapillary cells and a conspicuous infiltrate of polymorphonuclear leukocytes that characterize the disease by routine LM (Fig. 12A). Capillary walls were slightly to moderately thickened but showed no evidence of the lumps or bumps that would appear in upcoming electron micrographs. However, when the identical H\&E section was viewed by H\&E/FM, immune complex deposits were readily identified, many of them appearing as large, discrete, cone-shaped structures bulging from the outer surface of capillary walls (Fig. 12B). The combined use of LM and FM on the same H\&E section, therefore, helped to establish the diagnosis of postinfectious GN and presage the findings of immunofluorescence and EM.

\section{Other Cases}

H\&E/FM was not helpful in identification of glomerular diseases in which deposits were not formed as discrete bodies or aggregates. Diseases including dense-deposit disease, light chain deposition disease, amyloidosis, and fibrillary GN showed fluorescence of the GBM or mesangia that focally may have had greater intensity than normal but did not display a readily recognizable pattern to distinguish deposits from the surrounding matrix. For example, dense-deposit disease showed fluorescent ribbons by H\&E/FM that were of irregular thickness and of variably increased intensity (Fig. 13); but ribbons were obvious and recognized as significant only retrospectively once the diagnosis was known. Similarly, H\&E/FM did not significantly add to the study of other glomerular diseases also lacking formed deposits such as minimal change, diabetic nephropathy, microangiopathy, and thin membrane disease, although irregularities in the GBM may have been obvious again once the diagnosis was knowñ. H\&E/FM did not identify any differences in the glomerular capillary loops in antiGBM disease, although crescents were accentuated and fibrin deposits had a distinct orange tinge.

The hyalin-like deposits frequently seen in sclerosing glomerular diseases were greatly accentuated with H\&E/FM and identified as large nodular areas of fluorescence usually within focal glomerular segments exhibiting sclerosis by LM.
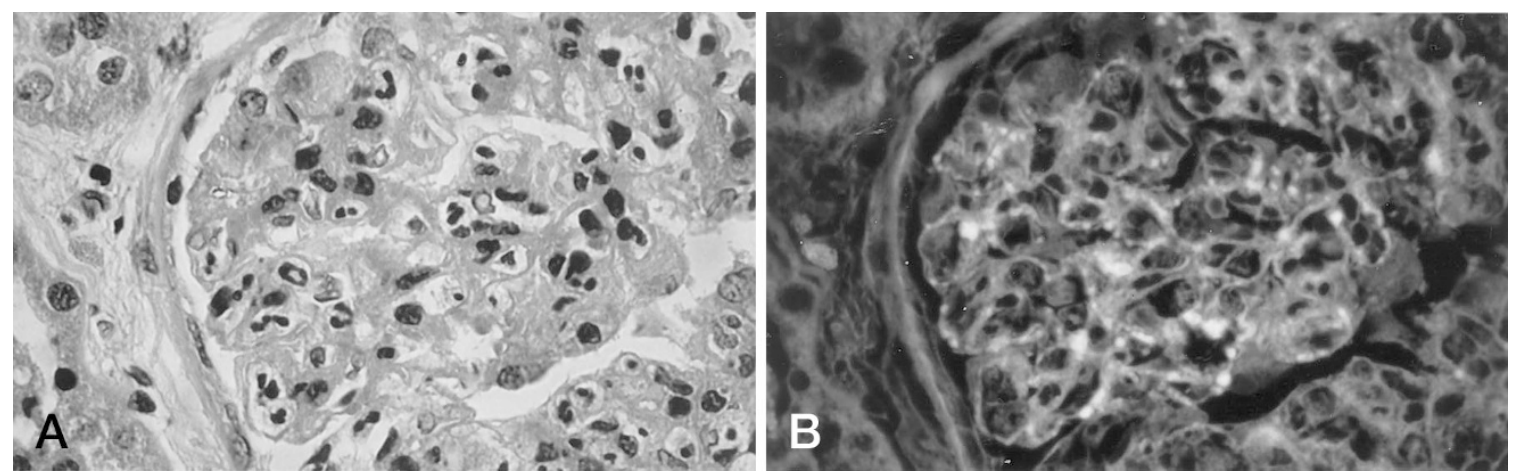

FIGURE 12. Comparison of routine light microscopy (LM; A) and fluorescence microscopy (FM; B) of identical hematoxylin-eosin stain (H\&E)stained section of postinfectious glomerulonephritis. By LM (A), there is glomerular lobular accentuation, endocapillary cell proliferation, and polymorphonuclear leukocytes but no evidence of immune complex deposits. By hematoxylin-eosin stain, viewed by FM (B), fluorescent deposits corresponding to immune complex deposits are seen bulging from outer surfaces of glomerular capillary loops $(460 \times)$. 


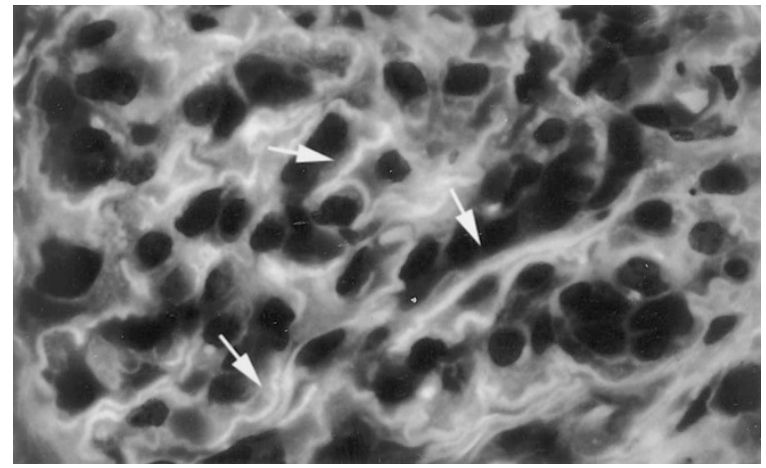

FIGURE 13. Hematoxylin-eosin stain, viewed by fluorescence microscopy, of dense-deposit disease. Fluorescent ribbons of variable thickness and intensity (arrows) are seen along glomerular capillary loops $(460 \times)$.

\section{Analysis of $\mathrm{H} \& \mathrm{E} / \mathrm{FM}$}

H\&E/FM appeared to significantly add to the light-microscopic recognition of known cases of renal glomerular diseases, especially immunecomplex glomerulonephritis. To test the validity of $\mathrm{H} \& \mathrm{E} / \mathrm{FM}$ as a prospective tool for the diagnosis of renal disease, 261 consecutive renal biopsies were examined by H\&E/FM without knowledge of results from immunofluorescence or EM. In this study, 202 biopsies were adequate for analysis. Fifty-nine were inadequate because of the presence of no glomeruli or only sclerotic glomeruli. One hundred thirty-one of the cases were unrelated to ICGN and did not show any H\&E/FM fluorescent deposits having recognizable GBM or mesangial patterns suggestive of immune complex glomerular disease. These nonICGN cases are shown in order of their total numbers in Table 2. Large fluorescent nodular deposits were identified in 12 non-ICGN cases including diabetic nephropathy (5), focal segmental glomerular sclerosis (6), and light chain nephropathy (1). These deposits did not have a specific pattern associated with ICGN and appeared related to hyaline-like granules in areas of segmental or total glomerular sclerosis.

There were 71 cases of ICGN. The results of $\mathrm{H} \& \mathrm{E} / \mathrm{FM}$ analysis of these cases are summarized in Table 3 and described in detail as follows. Of the 71 cases, 30 were of MGN including WHO Class V Lupus. Twenty-eight of the 30 membranous cases

TABLE 2. Non-ICGN Kidney Biopsy Cases in Order of Total Number

Focal segmental glomerular sclerosis Nonspecific changes (6) (31)

Tubular and/or interstitial disease (19)

Minimal change disease (15)

Diabetic nephropathy (15)

Transplant kidney (14)

Pauci-immune crescentic GN (9)

Glomerular sclerosis (7)

Thin membrane disease $(6)$

Microangiopathy (4)

Light chain disease (2)

Amyloid (1)

Myeloma (1)

Fibrillary glomerulonephritis (1)
(93\%) showed fluorescent deposits along the GBM with the characteristic beads-on-a-string pattern similar to those shown in Figures 3B, 6B, and 7. This pattern was seen only in cases of MGN. No other case of ICGN had this pattern; and this pattern was never seen in a non-ICGN case. Of the two MGN cases not showing beads on a string, one was scored as having equivocal fluorescent deposits along the GBM by H\&E/FM and was later shown to be a Stage I MGN with small plaque-like deposits in the subepithelial spaces by EM. The other had no discernible deposits by H\&E/FM and was later identified by EM as a resolved MGN having extensive replacement of electron-dense deposits by electron-lucent inclusions along the GBM.

Twenty cases were of MesGN, including IgA nephropathy/Henoch-Schönlein purpura (14), Class II lupus nephritis (4), IgM nephropathy (1), and MesGN not otherwise specified (NOS) (1) in which immunofluorescence was unavailable. Five of these 20 demonstrated definite mesangial deposits by $\mathrm{H} \& \mathrm{E} / \mathrm{FM}$, corresponding to prominent mesangial deposits by retrospective review of the EM and immunofluorescence. Five had equivocal mesangial deposits, and 10 showed no mesangial deposits by $\mathrm{H} \& \mathrm{E} / \mathrm{FM}$, corresponding to minute or sparse electron dense deposits by EM in 9 of these 15 combined cases. The other 6 of these 15 showed prominent deposits by EM. Two MesGN cases had H\&E/FM deposits in the GBM that were confirmed by EM, as well as H\&E/FM deposits in the mesangia.

Thirteen cases were of either MPGN I (9 cases) or diffuse proliferative lupus nephritis (WHO Class IV; 4 cases). Ten $(77 \%)$ of these cases showed definite GBM deposits by H\&E/FM appearing either granularly or as prominent confluent arclike fluorescent deposits conforming to the contour of the peripheral capillary loops. Only 3 showed definite mesangial deposits. Two cases had equivocal GBM deposits. By $\mathrm{EM}$, one of the equivocal cases was a resolving MPGN with flocculent, electron-lucent expansion of the GBM; the other had numerous small mesangial deposits and a few scattered GBM deposits. One case was scored as having no deposits by H\&E/FM and showed only minute deposits by EM.

Eight cases were reported as ICGN without further classification (NOS). Two of these had definite mesangial and/or GBM deposits by H\&E/FM that agreed well in size and distribution to electron dense deposits demonstrated by EM. Five showed equivocal H\&E/FM fluorescent deposits. Four of these equivocal cases showed either very small, tiny, or minute electron-dense deposits by EM that may have been too small to have been detected or discriminated from granularity of constituent mesangial components by H\&E/FM. The fifth case that 


\begin{tabular}{|c|c|c|c|c|c|c|}
\hline Diagnosis & $\begin{array}{l}\text { Significant } \\
\text { Findings by } \\
\text { H\&E/FM }\end{array}$ & $\begin{array}{c}\text { Positive } \\
\text { Findings by } \\
\text { H\&E/FM } \\
(n / N)\end{array}$ & $\begin{array}{l}\text { EM of Cases with Positive } \\
\text { Findings by H\&E/FM }\end{array}$ & $\begin{array}{c}\text { Equivocal } \\
\text { Findings by } \\
\text { H\&E/FM } \\
(n / N)\end{array}$ & $\begin{array}{c}\text { Negative } \\
\text { Findings by } \\
\text { H\&E/FM } \\
(n / N)\end{array}$ & $\begin{array}{l}\text { EM Results of Cases with } \\
\text { Equivocal or Negative Findings }\end{array}$ \\
\hline MGN & $\begin{array}{l}\text { Beads-on-a- } \\
\text { string } \\
\text { deposits }\end{array}$ & $28 / 30$ & $\begin{array}{l}\text { Subepithelial electron-dense } \\
\text { deposits }\end{array}$ & $0 / 30$ & $2 / 30$ & $\begin{array}{l}1 \text { with resolved deposits } \\
1 \text { with very small deposits }\end{array}$ \\
\hline MesGN & $\begin{array}{r}\text { Mesangial } \\
\text { deposits }\end{array}$ & $5 / 20$ & $\begin{array}{l}\text { Mesangial electron-dense } \\
\text { deposits }\end{array}$ & $5 / 20$ & $10 / 20$ & $\begin{array}{l}9 \text { with minute deposits } \\
6 \text { with prominent deposits }\end{array}$ \\
\hline MPGN & $\begin{array}{l}\text { Granular and } \\
\text { arc-like } \\
\text { deposits }\end{array}$ & $10 / 13$ & $\begin{array}{l}\text { Granular and confluent } \\
\text { subendothelial and } \\
\text { mesangial dense deposits }\end{array}$ & $2 / 13$ & $1 / 13$ & $\begin{array}{l}1 \text { with resolved deposits } \\
2 \text { with minute deposits }\end{array}$ \\
\hline $\begin{array}{l}\text { ICGN } \\
\text { NOS }\end{array}$ & $\begin{array}{l}\text { Mesangial } \\
\text { and/or GBM } \\
\text { deposits }\end{array}$ & $2 / 8$ & $\begin{array}{l}\text { Dense deposits with size } \\
\text { and distribution } \\
\text { compatible with H\&E/FM }\end{array}$ & $5 / 8$ & $1 / 8$ & $\begin{array}{l}5 \text { with minute deposits } \\
1 \text { with prominent deposits }\end{array}$ \\
\hline
\end{tabular}

H\&E/FM, hematoxylin and eosin stain viewed by fluorescence microscopy; EM, electron microscopy; MGN, membranous glomerulonephritis; MesGN, mesangiopathic glomerulonephritis; MPGN, membranoproliferative glomerulonephritis; ICGN NOS, immune complex glomerulonephritis not otherwise specified; GBM, glomerular basement membrane.

was equivocal by $\mathrm{H} \& \mathrm{E} / \mathrm{FM}$ had prominent dense deposits in the mesangia and GBM by EM. One case showed no fluorescent deposits and had only very few small deposits by EM.

\section{Hollande's Fixative versus Formalin}

Six cases of membranous glomerulonephritis (MGN) originally fixed in formalin were examined by H\&E/FM using microscopic conditions identical to those used in evaluation of Hollande's fixed tissue. Each case showed tissue fluorescence similar to that described in the Normal Kidney section, with the exception of variable thickening of peripheral capillary loops. There were no spêcific beadson-a-string patterns of fluorescence or other deposits suggestive of immune complex deposits similar to that seen in Hollande's fixed cases of MGN.

\section{DISCUSSION}

We have shown that fluorescence microscopy (FM) of routine $\mathrm{H} \& \mathrm{E}$-stained sections (H\&E/FM) of renal biopsies fixed in Hollande's fixative demonstrates histologic and pathologic structures not seen by standard light microscopy, including immune complex deposits in both glomerular basement membranes (GBM) and mesangia. We have also shown in a blind evaluation of 261 consecutive renal biopsies that these findings (summarized in Table 3) occurred reliably in a high enough percentage of cases of ICGN to make H\&E/FM of renal biopsies diagnostically useful. In all cases of ICGN, fluorescent deposits by H\&E/FM corresponded well to the size and distribution of deposits seen by immunofluorescence and EM. The absence of falsepositive findings in cases having negative immunofluorescence and EM (e.g., minimal change, tubular/interstitial disease, thin GBM) supported the reliability of $\mathrm{H} \& \mathrm{E} / \mathrm{FM}$.

\section{Membranous Glomerulonephritis}

In MGN (including lupus nephritis WHO Class V), H\&E/FM demonstrated brightly fluorescing deposits along glomerular capillary loops in a pattern appearing as a beads on a string, in which beads corresponded to immune complex deposits linearly strung along glomerular basement membranes.

In our group of 30 MGN cases, 28 showed positive beads on a string, including 4 cases of MGN Stage I. Of the two cases that did not show beads on a string, one was a MGN Stage I with minute plaquelike deposits by EM, which may have been beneath the detection limits of H\&E/FM. The other was a resolved MGN with electron lucent deposits by EM, which may be unsuitable for H\&E/FM demonstration. The combined facts that (1) there were never false-positive beads-on-a-string; (2) $>93 \%$ of MGN cases showed beads on a string; and (3) beads on a string were never seen in ICGN cases other than MGN make the finding of a beads-on-a-string pattern of fluorescence highly sensitive and specific for MGN.

\section{for}

\section{Mesangiopathic Glomerulonephritis}

Of 20 MesGN (immune complex type) cases, only 5 were unequivocally positive using H\&E/FM, with distinct mesangial fluorescence corresponding to prominent mesangial deposits by EM and immunofluorescence. The other 15 had either equivocal or negative findings by H\&E/FM even though some of those cases had mesangial deposits that were conspicuous by immunofluorescence and/or EM. The difficulty in identifying mesangial deposits by H\&E/FM, even when prominent by immunofluorescence and EM, perhaps lies in the complexity of the mesangia in MesGN. In many cases of MesGN, pathologic changes included a mixture of immune complex deposits, cellularity that may have been proliferated, and expanded matrix with variable 
density that confounded the ability to distinguish mesangial components. Although positive identification of mesangial deposits by H\&E/FM was possible in some cases, H\&E/FM did not appear reliable enough to identify all cases of MesGN, even those having prominent mesangial deposits.

\section{Membranoproliferative Glomerulonephritis}

In renal cases having cellular proliferation, lobular accentuation, mesangial expansion, and loss of capillary patency, MPGN (including lupus nephritis WHO Class IV and cryoglobulinemic GN) was high on the suspicion list, and H\&E/FM deposits were largely anticipated. In our series, there were 13 cases of MPGN with 10 having large fluorescent granular or continuous deposits conforming to the outlines of glomerular capillary loops. The absence of loop deposits in 3 cases was attributed to small size or resolution of deposits as demonstrated by EM. Only 3 showed mesangial deposits; but mesangial deposits, as in MesGN, were frequently difficult to identify by H\&E/FM. Overall, the demonstration of deposits in MPGN was expected and subsequently realized, thereby further demonstrating the usefulness of H\&E/FM in renal pathology.

\section{Fluorescence of H\&E Sections}

Fluorescence of hematoxylin and eosin-stained sections depends on the eosin component, which is commercially produced by bromination of fluorescein. A previous report demonstrated fluorescence of H\&E-stained frozen sections of renal biopsies but stated that the method was not sensitive enough to reveal subtle GBM changes or small immune deposits (9). In our report, we showed small immune deposits in H\&E-stained permanent sections of Stage I-II membranous glomerulonephritis and larger deposits in a variety of ICGN cases. Whereas the other investigators described fluorescence of frozen sections only, their protocol included formalin-fixed tissues for light microscopy. We assume that they must have tried but failed to demonstrate immune complex deposits using fluorescence microscopy on their H\&E-stained sections of paraffin-embedded, formalin-fixed tissue.

We have demonstrated immune complex deposits using H\&E/FM on permanent sections of renal biopsies fixed in Hollande's fixative. But, as assumed for the previous investigators, we were unable to detect immune deposits or show a beadson-a-string pattern of fluorescence in six known cases of membranous glomerulonephritis that were fixed in formalin. We therefore conclude that our use of Hollande's fixative was critical for the demonstration of deposits.
Hollande's is a formalin-based fixative. But there are components of Hollande's, not present in routine formalin fixatives, that may permit increased deposition of eosin at the sites of immune complex deposits in histologic sections, which when viewed using $\mathrm{H} \& \mathrm{E} / \mathrm{FM}$ have areas of increased fluorescence marking the location of deposits. Picric acid is a well-known dye and is a component of a number of histologic fixatives including Bouin's and Hollande's. It reacts with basic proteins to form crystalline picrates that can be replaced by other acid dyes including eosin (10). By reacting with proteinaceous immune complex deposits, picric acid may facilitate eosin deposition during subsequent staining processes, leading to enhanced localized fluorescence using H\&E/FM. Furthermore, Hollande's is known to induce clumping of nuclear chromatin $(7,8)$, which gives H\&E-stained tissue sections such good nuclear detail. Picric acid and glacial acetic acid components of Hollande's may also induce denaturation and clumping of immune complex proteins that does not normally occur with fixation in neutral buffered formalin. Such clumping would permit better eosin staining and easier visualization of immune deposits using H\&E/FM. Copper acetate was originally added to Hollande's to improve the dissolution of picric acid (11) but also was found to stabilize red blood cell membranes and certain cytoplasmic granules (8). Copper acetate may also act in a way similar to copper sulfate, which has been used as a mordant to promote color uptake in the textile dye industry. When used as part of the formulation of Hollande's, these components (picric acid, acetic acid, and copper acetate) may enhance the eosinophilia of proteinaceous immune complex domains within the mucopolysaccharide environment of the GBM and mesangial matrix. A localized enhancement in eosinophilia would produce an H\&E/FM image with increased fluorescence at the site of immune complex deposits, thereby allowing easier recognition of immune complex glomerulonephritis at the lightmicroscopic level.

\section{CONCLUSION}

The standard for diagnostic evaluation of renal biopsies includes light-microscopic, immunofluorescence, and electron-microscopic studies. Although each is necessary for complete characterization of renal disease, it is important that maximum information be derived from the biopsy at each level of study. With H\&E/FM of Hollande's fixed kidney tissue, pathologists have an entirely new approach to the light-microscopic evaluation of renal biopsies with results leading to the confident identification of cases of ICGN in advance of 
both immunofluorescence and EM. H\&E/FM, however, is not intended to replace or supersede any other accepted technique or protocol established for the study of renal biopsies.

Acknowledgments: We thank Charles J. Nicely, M.D., Riverside Methodist Hospital (Columbus, OH) for supplying $H \& E$ slides of formalin-fixed cases of membranous glomerulonephritis.

\section{REFERENCES}

1. Jones DB. Nephrotic glomerulonephritis. Am J Clin Pathol 1975;63:310-7.

2. Cohen AH. Masson's trichrome stain in evaluation of renal biopsies. An appraisal. Am J Clin Pathol 1976;65:631-43.

3. Ehrenreich T, Espinosa T. Chromotrope silver methenamine stain of glomerular lesion. Am J Clin Pathol 1971;56:448-51.
4. Gamble CN. Periodic acid-Schiff-light green stain to detect glomerular protein deposits by routine light microscopy. Am J Clin Pathol 1975;63:310-7.

5. Mazzucco G, Basolo B, Monga G. The use of Mallory's phosphotungstic acid-hematoxylin (PTAH) stain in renal pathology. Pathol Res Pract 1982;175:380-91.

6. Mihatsch MJ, Bremer J. Acid fuchsin orange G-stain (AFOG) for glomerular protein deposits [letter]. Clin Nephrol 1978; 9:259.

7. Murphy WM, Ramsey J, Soloway MS. A better nuclear fixative for diagnostic bladder and prostate biopsies. J Urol Pathol 1993;1:79-87.

8. Haggitt RC. Handling of gastrointestinal biopsies in the surgical pathology laboratory. Lab Med 1982;13:272-8.

9. Bonsib SM, Reznicek M. Renal biopsy frozen section: a fluorescent study of hematoxylin and eosin-stained sections. Mod Pathol 1990;3:204-10.

10. Hopwood D. Fixation and fixatives. In: Bancroft JD, Stevens A, editors. Theory and practice of histological techniques. 2nd ed. New York: Churchill Livingston; 1982. p. 20-40.

11. Hollande AC. Enrichissement du liquide de Bouin en acide picrique, par addition d'acetate neutre de cuivre. Comptes Rendus Soc Biol 1918;LXXXI:17-20.
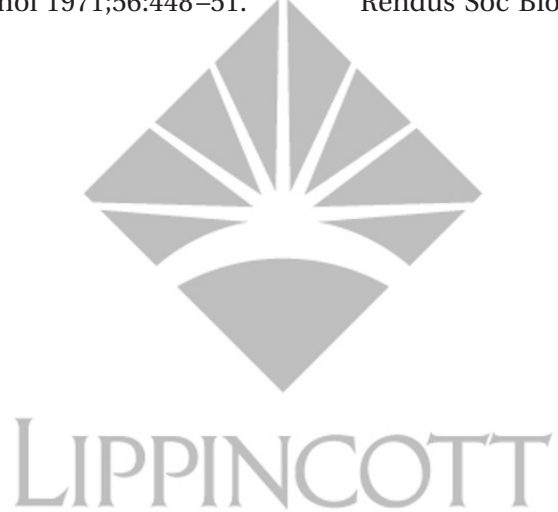

WILLIAMS \& WILKINS

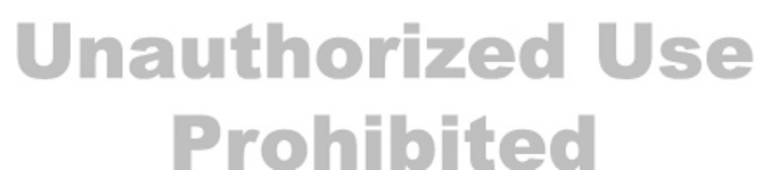

